\title{
Cyclic Einstein-Podolsky-Rosen steering
}

\author{
István Márton $\odot,{ }^{1, *}$ Sándor Nagy, ${ }^{2, \dagger}$ Erika Bene, ${ }^{1,+}$ and Tamás Vértesi $\odot^{1, \S}$ \\ ${ }^{1}$ MTA Atomki Lendület Quantum Correlations Research Group, Institute for Nuclear Research, P.O. Box 51, H-4001 Debrecen, Hungary \\ ${ }^{2}$ Department of Theoretical Physics, University of Debrecen, P.O. Box 5, H-4010 Debrecen, Hungary
}

(Received 23 June 2021; accepted 8 October 2021; published 8 November 2021)

\begin{abstract}
Einstein-Podolsky-Rosen (EPR) steering is a form of quantum correlation that exhibits a fundamental asymmetry in the properties of quantum systems. Given two observers, Alice and Bob, there are known to exist bipartite entangled states which are one-way steerable in the sense that Alice can steer Bob's state, but Bob cannot steer Alice's state. Here, we generalize this phenomenon to three parties and find a cyclic property of tripartite EPR steering. In particular, we identify a three-qubit state whose reduced bipartite states are one-way steerable for arbitrary projective measurements. Moreover, the three-qubit state has a cyclic steering property in the sense that by arranging the system in a triangular configuration the neighboring parties can steer each other's states only in the same (e.g., clockwise) direction. That is, Alice can steer Bob's state, Bob can steer Charlie's state, and Charlie can steer Alice's state, but not the other way around.
\end{abstract}

DOI: 10.1103/PhysRevResearch.3.043100

\section{INTRODUCTION}

Quantum entanglement is a remarkable phenomenon without counterpart in classical physics [1,2]. Notably, it gives rise to nonlocal correlations between distant particles as was pointed out by Einstein, Podolsky, and Rosen (EPR) [3]. Later, Bell [4] proved that nonlocality is inherent to quantum theory. Today, Bell nonlocality is considered a fundamental feature of the theory and plays an important role in quantum information processing $[1,5,6]$.

The concept of steering (also known as EPR steering) was proposed by Schrödinger in 1935 [7], which concept brought novel insight to the study of nonlocal correlations $[8,9]$. Consider two distant observers-say, Alice and Bob-who share a pair of two spin-(1/2) particles in the maximally entangled singlet state

$$
\left|\psi_{-}\right\rangle=\left(|0\rangle_{A}|1\rangle_{B}-|1\rangle_{A}|0\rangle_{B}\right) / \sqrt{2}
$$

Alice can steer the state of Bob's system by performing a measurement on her system. In particular, if Alice projects by measuring her share of the state into the state

$$
\left|v_{A}\right\rangle=a|0\rangle+b|1\rangle,
$$

Bob's system immediately collapses to the orthogonal state

$$
\left|v_{B}\right\rangle=-b^{*}|0\rangle+a^{*}|1\rangle,
$$

\footnotetext{
*marton.istvan@atomki.hu

†sandor.nagy@ science.unideb.hu

*bene@atomki.hu

§tvertesi@atomki.hu
}

Published by the American Physical Society under the terms of the Creative Commons Attribution 4.0 International license. Further distribution of this work must maintain attribution to the author(s) and the published article's title, journal citation, and DOI. where $*$ means complex conjugation. Note that due to normalization $a a^{*}+b b^{*}=1$, the coefficients $a$ and $b$ of Bob's state can be given explicitly by two angles $\theta$ and $\varphi$ :

$$
\begin{aligned}
& a=\cos (\theta / 2), \\
& b=\sin (\theta / 2) \exp (i \varphi),
\end{aligned}
$$

where $a$ is real valued, since a global phase of the state (2) is unobservable. The two angles $\theta$ and $\varphi$ can be adjusted to arbitrary values by Alice by performing a well-chosen measurement on her system. Hence Alice can prepare different states for Bob; that is, she can steer Bob's state.

Originally, EPR steering was studied in the context of continuous variable systems $[10,11]$; however, the effect was soon formalized by Wiseman et al. [12] as a quantum information task for general multipartite systems. EPR steering can also be seen as a form of quantum correlation that is intermediate between entanglement and Bell nonlocality $[12,13]$. To illustrate these properties, let us consider the two-qubit singlet state (1). By adding some white noise to it, we obtain the one-parameter family of two-qubit Werner states [14]

$$
\rho_{W}(p)=p\left|\psi_{-}\right\rangle\left\langle\psi_{-}\right|+(1-p) \mathbb{I}_{4} / 4,
$$

where $(1-p) \in[0,1]$ is a noise parameter. One can now ask about the critical limit $p$, above which the state (4) is entangled, EPR steerable, and Bell nonlocal for arbitrary projective measurements. We list below the three different cases.

(i) The state (4) is entangled if and only if $p>1 / 3$. This can be directly seen by using Peres's positivity of partial transpose (PPT) criterion [15].

(ii) The state (4) is EPR steerable if and only if $p>1 / 2$ as shown by Werner [14] and Wiseman et al. [12].

(iii) The state (4) is Bell nonlocal for $p>0.6964$ [16] and admits a local hidden variable model for $p \leqslant 0.6829$ [17]. These bounds arise from a connection to the Grothendieck constant of order 3 [18]. 
The above nonoverlapping bounds show that entanglement, steering, and Bell nonlocality are different under projective measurements. However, the more general case of positive-operator-valued measure (POVM) measurements was also considered in Ref. [19], and the same conclusion has been drawn in this case as well.

Steering finds applications in quantum information tasks, such as quantum key distribution [20,21], randomness generation [22-24], and channel discrimination [25]. More recently, it has also been linked to quantum metrology [26].

Experimental investigations have been reported [27-29]; in particular, the steerability property of the family of two-qubit states (4) has been analyzed in detail [13]. In addition, steering has been used as a tool for detecting entanglement in BoseEinstein condensates [30-32] and atomic ensembles [33]. Notably, in 2012, a loophole-free EPR-steering experiment was performed [27] (see also Ref. [34]). We note that the more recent loophole-free Bell experiments [35] also demonstrate loophole-free EPR steerability.

A distinctive feature of EPR steering is the asymmetry between the roles of observers $[12,36]$. In particular, this asymmetry is not present in the phenomenon of entanglement and Bell nonlocality. A steering test can be understood as the task of distributing entanglement from an untrusted Alice to a trusted Bob, a task formalized in Ref. [12]. Concretely, consider two particles in different locations, which are controlled by Alice and Bob. Alice tries to convince Bob that they share an entangled state $\rho_{A B}$ of these two particles. Bob, however, does not trust Alice, and therefore asks her to steer the state of his particle using different measurements. Suppose that Alice can choose to perform $m$ different measurements labeled by $x=(1, \ldots, m)$ on her particle. Denote the POVM elements of her outcome $a$ for a given setting $x$ by $M_{a \mid x}$. These POVM elements satisfy $M_{a \mid x} \geqslant 0$ for each choice of $x$ and outcome $a$, and we also have $\sum_{a} M_{a \mid x}=\mathbb{I}_{d}$, where $d$ is the dimension of the Hilbert space of Alice's subsystem. We will focus primarily on two-qubit states $\rho_{A B} \in L\left(\mathbb{C}^{2} \otimes \mathbb{C}^{2}\right)$ shared by Alice and Bob, and on nondegenerate projective measurements $M_{a \mid x}$ for Alice, which can be written in the following form:

$$
\begin{aligned}
& M_{0 \mid x}=\left|v_{A, x}\right\rangle\left\langle v_{A, x}\right|, \\
& M_{1 \mid x}=\mathbb{I}_{2}-\left|v_{A, x}\right\rangle\left\langle v_{A, x}\right|,
\end{aligned}
$$

where $\left|v_{A, x}\right\rangle$ has the form (2), that is $\left|v_{A, x}\right\rangle=a_{x}|0\rangle+b_{x}|1\rangle$. The set of conditional states $\left\{\rho_{a \mid x}\right\}$ that Alice can prepare for Bob by measuring $x$ and obtaining $a$ forms the so-called steering assemblage. This set is given by the formula

$$
\rho_{a \mid x}=\operatorname{tr}_{A}\left(M_{a \mid x} \otimes \mathbb{I} \rho_{A B}\right),
$$

where

$$
p(a \mid x)=\operatorname{tr}\left(\rho_{a \mid x}\right)
$$

is the probability that Alice obtains outcome $a$ for her setting $x$. Note that the states (5) are subnormalized in general; that is, $\operatorname{tr}\left(\rho_{a \mid x}\right)<1$, but $\sum_{a} \operatorname{tr}\left(\rho_{a \mid x}\right)=1$ holds true for all $x$. In particular, the assemblage (5) carries all the information about the EPR-steering setup.

We say that a state $\rho_{A B}$ demonstrates steering from Alice to Bob (or put differently, Alice can steer the state of Bob) if Bob's assemblage (5) cannot be written in the so-called local hidden state (LHS) form [12]:

$$
\rho_{a \mid x}=\sum_{\lambda} \omega(\lambda) p_{\lambda}(a \mid x) \rho_{\lambda},
$$

where $\lambda$ represents a classical random variable known to $\mathrm{Al}$ ice with an arbitrary probability distribution $\omega(\lambda)$. That is, we have $\sum_{\lambda} \omega(\lambda)=1$ and $\omega(\lambda) \geqslant 0$ for all $\lambda$. Note that (7) defines a so-called local hidden state strategy. In fact, the assemblage (7) can be prepared by Alice and Bob without sharing an entangled quantum state: Shared randomness (characterized by the variable $\lambda$ and Bob's qubit states $\rho_{\lambda}$ ) is sufficient to produce the assemblage (7).

Hence, in the steering problem, Bob's task is to determine whether the states $\rho_{a \mid x}$ in the assemblage (5) admit a decomposition of the form (7). If this is the case, then Bob will not be convinced that entanglement is present. Conversely, if it can be shown that the assemblage (5) cannot be written in the form (7), then this indicates the presence of entanglement, and we say that the state $\rho_{A B}$ is steerable. This steerability can be conveniently proved using the so-called EPR-steering inequalities (which we discuss in our first scenario).

Let us also remark that a decomposition of the form (7) for a given finite number of settings $m$ does not imply that the underlying state $\rho_{A B}$ is unsteerable. It can well be the case that as the number of settings $m$ increases, the assemblage (5) can be no longer written in the LHS form (7) and thus becomes steerable. Hence, more generally, we say that a state $\rho_{A B}$ is unsteerable from Alice to Bob if the assemblage $\left\{\rho_{a \mid x}\right\}$ in Eq. (5) admits a decomposition of the form (7) for all possible measurements $M_{a \mid x}$. That is, Alice in general has to consider an infinite number of measurement settings $x$. We will mainly focus on projective measurements, in which case we say that the state $\rho_{A B}$ is unsteerable from Alice to Bob for projective measurements.

We also note that the above definition of EPR steering treats the roles of the two observers differently. The question, already raised in Ref. [12], is whether there exists a bipartite entangled state $\rho_{A B}$ such that Alice can steer Bob's state, but Bob cannot steer Alice's state. This phenomenon, which has been called one-way steering, was first investigated theoretically in continuous variable systems for a restricted class of measurements [37,38]. Then a simple class of one-way steerable two-qubit states was found for projective measurements [36]. This phenomenon was further studied in other two-qubit systems [39-41]. Finally, the problem was settled by finding a one-way steerable two-party state for the most general POVM measurements [19]. On the experimental side, early examples for one-way steering were presented for continuous variable systems involving Gaussian measurements [42]. On the other hand, for discrete systems, one-way steering including the general case of POVMs was first demonstrated experimentally by Wollmann et al. [43].

Let us mention that the study of steering is not restricted to the bipartite case. Indeed, multipartite steering can be viewed as a semi-device-independent task [44], where some of the parties are trusted and some of them are untrusted. In this scenario, for instance, genuine tripartite entanglement $[1,2,45]$ can be detected through the phenomenon of EPR steering [46]. Furthermore, monogamy relations have 


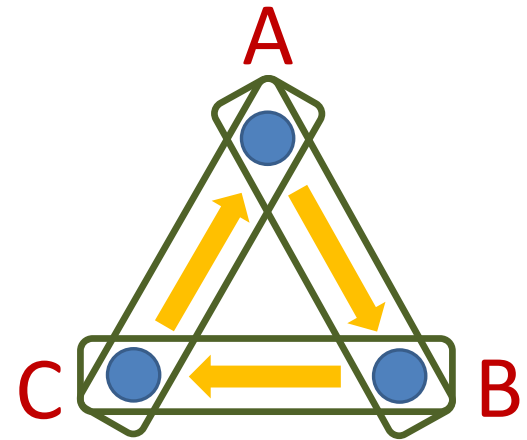

FIG. 1. Setup for cyclic EPR steering. The figure depicts a three-qubit state with its two-party reduced states shown as green rectangles. The yellow arrows indicate that steering takes place in the clockwise direction. That is, steering occurs from $A$ to $B$, from $B$ to $C$, and from $C$ to $A$. However, steering is not possible in the other (anticlockwise) direction, even if the untrusted party can perform arbitrary projective measurements.

also been studied in the context of steering for three-qubit systems [47].

\section{OUR MAIN RESULT}

We present a class of three-qubit entangled states which exhibits a cyclic steering property of quantum correlations. Specifically, we consider a three-qubit state in a triangular configuration shared by three partners, Alice, Bob, and Charlie, which state has the following property (see also Fig. 1): If any qubit is removed from the tripartite system, the remaining two-qubit state is one-way steerable. Let us denote by $P_{1}$ and $P_{2}$ the two parties left, and by $\rho_{P_{1} P_{2}}$ their respective reduced two-qubit state. Let us use the notation $P_{1} \rightarrow P_{2}$ if the state $\rho_{P_{1} P_{2}}$ demonstrates steering from $P_{1}$ to $P_{2}$, but it does not demonstrate steering from $P_{2}$ to $P_{1}$ considering arbitrary projective measurements (the case of POVM measurements and higher-dimensional systems is briefly discussed later).

In our particular case we prove the following steering properties of the $A-B-C$ tripartite system (see Fig. 1): $A \rightarrow B$, $B \rightarrow C$, and $C \rightarrow A$. To this end, we consider a translationally invariant three-qubit state $\rho_{A B C}$; that is, we have $\rho_{A B C}=$ $S \rho_{A B C} S^{\dagger}$, where $S$ is the right-shift operator:

$$
S=\sum_{i, j, k=0}^{1}|i j k\rangle\langle j k i| .
$$

This state has two-qubit reduced states with the following property:

$$
\rho_{A B}=\rho_{B C}=\rho_{C A},
$$

where $\rho_{A B}=\operatorname{tr}_{C}\left(\rho_{A B C}\right)$ denotes the two-qubit reduced state of Alice and Bob. If we swap Alice and Bob,

$$
\rho_{B A}=V \rho_{A B} V^{\dagger},
$$

where $V$ is the two-qubit flip operator, we do not usually have $\rho_{A B}=\rho_{B A}$. However, this property holds true for permutationally invariant three-qubit states $\rho_{A B C}$, such as the famous W [48] and Greenberger-Horne-Zeilinger (GHZ) states [49].
With the above symmetry of the state, $\rho_{A B C}=S \rho_{A B C} S^{\dagger}$, our task then boils down to finding a translationally invariant three-qubit state $\rho_{A B C}$ for which the two-qubit reduced state $\rho_{A B}$ is one-way steerable. That is, the state $\rho_{A B}$ does not demonstrate steering from Bob to Alice for any set of projective measurements of Bob. However, the state $\rho_{A B}$ is steerable from Alice to Bob, i.e., it does violate a specific steering inequality with well-chosen measurements of Alice. The proof of the existence of such a state $\rho_{A B C}$ will be based on the recent geometrical approach of Nguyen et al. [41].

The structure of this section is as follows. Our starting point is the construction of a family of translationally invariant three-qubit states $\rho_{A B C}$. With these states in hand, we solve the above-stated problem in two steps. (i) As a preliminary step, we first find a state from this family for which the two-qubit marginal $\rho_{A B}$ does violate a six-setting steering inequality. However, the swapped two-qubit state $\rho_{B A}$ does not violate the same steering inequality considering arbitrary projective measurements. This result already proves an asymmetric property of the two-qubit marginal $\rho_{A B}$, although it does not yet solve the problem that we originally formulated. (ii) We present a translationally invariant three-qubit state $\rho_{A B C}$ for which the reduced states $\rho_{A B}=\rho_{B C}=\rho_{C A}$ are one-way steerable for projective measurements.

Finally, we present a different construction of cyclic EPRsteering states, which makes use of one-way steerable states known from the literature. This construction allows us to prove the existence of three-party cyclic steerable states for the most general POVM measurements. However, in this case the minimum dimension of the component space of the tripartite quantum state is at least four. To demonstrate the properties associated with steps (i) and (ii), a class of threequbit states is crucial, the construction of which is discussed below.

\section{A. The state}

We consider a scenario featuring three remote parties, Alice, Bob, and Charlie, who share the following state:

$$
\rho(p)=\frac{p}{3} \sum_{i=1}^{3}\left|\psi_{i}\right\rangle\left\langle\psi_{i}\right|+(1-p) \mathbb{I}_{8} / 8,
$$

where $\left|\psi_{1}\right\rangle$ is a generic pure three-qubit state

$$
\left|\psi_{1}\right\rangle=\sum_{i, j, k=0}^{1} \alpha_{i j k}|i\rangle_{A}|j\rangle_{B}|k\rangle_{C}
$$

and the other two states, $\left|\psi_{2}\right\rangle$ and $\left|\psi_{3}\right\rangle$, are related to $\left|\psi_{1}\right\rangle$ as follows:

$$
\left|\psi_{(n+1) \quad(\bmod 3)}\right\rangle=S^{n}\left|\psi_{1}\right\rangle
$$

for $n \geqslant 1$, where $S$ is the right-shift operator defined by (8). Observe the cyclic property $\left|\psi_{1}\right\rangle=S^{3}\left|\psi_{1}\right\rangle$ and the translational invariance of the three-qubit state (11). We also emphasize that $\rho(p)$ is completely defined by the pure state $\left|\psi_{1}\right\rangle$ and the noise parameter $(1-p)$. With the above definition of the state, we then proceed to our first scenario. 


\section{B. Scenario 1}

Here, we present a bipartite steering inequality and show its violation using the reduced two-qubit marginals of the state (11) and specific measurements $M_{a \mid x}$ for Alice. Note that Alice's \pm 1 observables are defined by $A_{x}=M_{0 \mid x}-M_{1 \mid x}$. Our steering inequality takes the following form:

$$
\sum_{x=1}^{m} \sum_{a=0}^{1} \operatorname{tr}\left(F_{a \mid x} \rho_{a \mid x}\right) \leqslant L,
$$

where $L$ is the maximum for the left-hand-side functional to be obtained with an assemblage of the LHS form (7). Violation of this inequality proves that the steering assemblage (5) cannot be reproduced by an LHS model (7). Let us use the following functional given by Saunders et al. [13] for (14):

$$
F_{a \mid x}=(-1)^{a} B_{x},
$$

where the traceless $2 \times 2$ Hermitian matrices $B_{x}$ are $B_{x}=$ $\vec{b}_{x} \cdot \vec{\sigma}$, where $\vec{b}_{x}, x=(1, \ldots, m)$ are some unit vectors and $\vec{\sigma}=\left(\sigma_{x}, \sigma_{y}, \sigma_{z}\right)$ denotes the vector of Pauli matrices. The maximum value $L$ can be obtained by solving the following integer programming problem:

$$
L=\max _{a_{x}= \pm 1}\left[\lambda_{\max }\left(\sum_{x=1}^{m} a_{x} B_{x}\right)\right]=\max _{a_{x}= \pm 1}\left\|\sum_{x=1}^{m} a_{x} \vec{b}_{x}\right\|,
$$

where $\|\vec{v}\|$ stands for the Euclidean norm of vector $\vec{v}$ and $\lambda_{\max }(X)$ denotes the largest eigenvalue of $X$. So, we have to consider a total of $2^{m}$ strings $\left[a_{1}, a_{2}, \ldots, a_{m}\right]$ (where $a_{x}=$ $\pm 1, x=1, \ldots, m)$ for Alice to obtain $L$. For small $m$ (e.g., for $m<20$ ), this task can be solved on a desktop computer by an exhaustive enumeration of all possible strings.

On the other hand, we use the formula (5) for $\rho_{a \mid x}$ to compute the maximum quantum value of the left-hand-side expression in Eq. (14) for fixed $\rho_{A B}$ and $B_{x}$ matrices. We then use $A_{x}=M_{0 \mid x}-M_{1 \mid x}$ to obtain

$$
Q\left(\rho_{A B}\right) \equiv \max _{-\mathbb{I} \leqslant A_{x} \leqslant \mathbb{I}} \sum_{x=1}^{m} \operatorname{tr}\left(A_{x} \otimes B_{x} \rho_{A B}\right) .
$$

The above optimization task (17) can be carried out for each $x$ separately. Indeed, let us write

$$
\operatorname{tr}\left(A_{x} \otimes B_{x} \rho_{A B}\right)=\operatorname{tr}\left(A_{x} G_{x}\right),
$$

where we defined

$$
G_{x}=\operatorname{tr}_{B}\left(\mathbb{I} \otimes B_{x} \rho_{A B}\right),
$$

which results in

$$
Q=\max _{-\mathbb{I} \leqslant A_{x} \leqslant \mathbb{I}} \operatorname{tr}\left(A_{x} G_{x}\right)=\operatorname{tr} \sqrt{G_{x} G_{x}^{\dagger}},
$$

i.e., the maximum is given by the trace norm of $G_{x}$, where the optimal observables of Alice are

$$
A_{x}=\sum_{i=1,2} \operatorname{sgn}\left(\lambda_{x, i}\right)\left|v_{x, i}^{A}\right\rangle\left\langle v_{x, i}^{A}\right|,
$$

where the eigendecomposition of $G_{x}$ is given as $G_{x}=$ $\sum_{i=1,2} \lambda_{x, i}\left|v_{x, i}^{A}\right\rangle\left\langle v_{x, i}^{A}\right|$.

Let us now present our results for the steering inequality (14) using the functional (15). Namely, we choose $B_{x}=$ $\vec{b}_{x} \cdot \vec{\sigma}$, in which case $B_{x}$ can be interpreted as Bob's observables in Eq. (17). In particular, we consider measurement

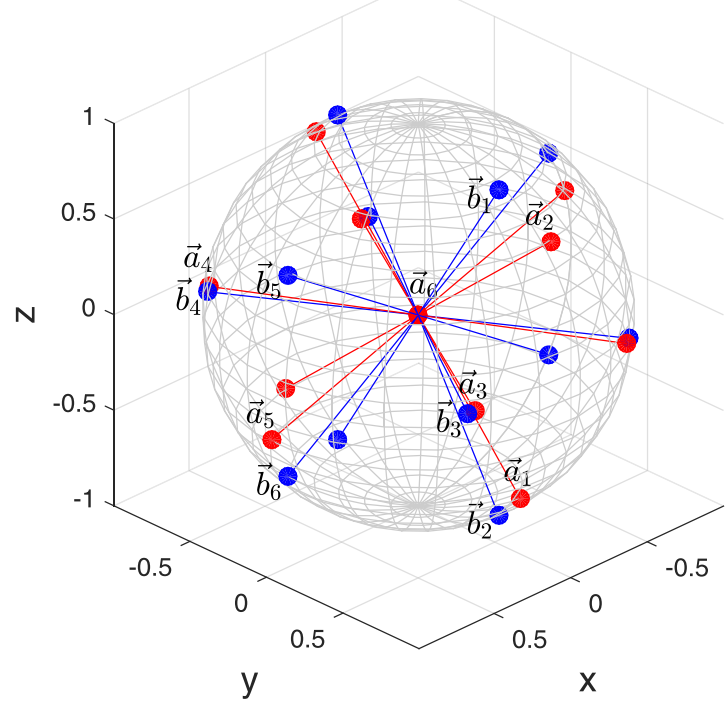

FIG. 2. Plot for the Bloch vectors of Alice's and Bob's measurements in EPR-steering scenario 1. Each measurement axis is defined by a pair of circles. The red circles correspond to Alice's Bloch vectors $\pm \vec{a}_{x}$, and the blue circles correspond to Bob's Bloch vectors $\pm \vec{b}_{x}$. Note that $\vec{a}_{6}$ is the zero-length vector corresponding to a degenerate measurement.

settings for Bob based on the regular icosahedron that have 12 vertices with six antipodal pairs $\pm \vec{b}_{x}, x=1, \ldots, 6$. The endpoints of the vectors $\vec{b}_{x}$ are marked in blue in Fig. 2. The coordinates of the vertices are given by the formulas (A1) in Appendix A.

Defining Bob's observables $B_{x}=\vec{b}_{x} \cdot \vec{\sigma}$ by the six icosahedral unit vectors $\vec{b}_{x}$, we get the following maximum for $L$ in Eq. (16):

$$
L=\left\|\sum_{x=1}^{6} a_{x} \vec{b}_{x}\right\|=\|v / n\|=1+\sqrt{5} \simeq 3.2361,
$$

where the vector $v=[2+2 \phi, 0,-2 \phi]$. Above, $\phi=(1+$ $\sqrt{5}) / 2$ is the golden ratio, and $n=\sqrt{1+\phi^{2}}$ is a normalization constant. Alice's corresponding strategies are $a_{1}=-1$ and $a_{x}=1$ for $x=(2, \ldots, 6)$.

Our goal now is to find the reduced state $\rho_{A B}$ of the translationally invariant state (11) such that

$$
\begin{aligned}
& Q\left(\rho_{A B}\right)>L, \\
& Q\left(\rho_{B A}\right) \leqslant L,
\end{aligned}
$$

where $\rho_{A B}=\operatorname{tr}_{C} \rho(p)$, with $\rho(p)$ given by (11), and the swapped state $\rho_{B A}$ is given by (10). Note that for a permutationally invariant (PI) state $\rho_{A B C}$, we have $\rho_{A B}=\rho_{B A}$ and both conditions in Eq. (22) cannot be fulfilled simultaneously. Therefore we need to guarantee that $\rho(p)$ is not PI. We will perform a heuristic search in order to find a feasible $\rho_{A B}$ in Eq. (22). To do so, we transform the feasibility problem (22) into a constrained optimization task:

$$
\begin{aligned}
\max & Q\left(\rho_{A B}\right) \\
\text { such that } & Q\left(\rho_{B A}\right) \leqslant L,
\end{aligned}
$$


where we set $p=1$ in Eq. (11). Hence, in the above task the only optimization variables are the $\alpha_{i j k}$ coefficients of $\left|\psi_{1}\right\rangle$ in Eq. (12). In addition, we set $\alpha_{111}=0$ and restrict the search to real-valued coefficients $\alpha_{i j k}$. One variable is spared due to the normalization condition $\sum_{i, j, k} \alpha_{i j k}^{2}=1$, leaving $2^{3}-2=$ 6 optimization variables.

To solve the above task, we use the Nelder-Mead simplex algorithm [50] by replacing the problem with an unconstrained problem, where we subtract a penalty term from the objective function $Q\left(\rho_{A B}\right)$. Specifically, we maximize the following objective value:

$$
q=Q\left(\rho_{A B}\right)-c \max \left[0, L-Q\left(\rho_{B A}\right)\right]
$$

over the $\alpha_{i j k}$ coefficients of $\left|\psi_{1}\right\rangle$. We set $c=2$ in the course of optimization, and $\max \left[x_{1}, x_{2}\right]$ gives the larger value between $x_{1}$ and $x_{2}$. The algorithm was run 500 times, and a couple of times we found the following (presumably) best objective $q=3.2688$ where $Q\left(\rho_{A B}\right)=3.2688$ and $Q\left(\rho_{B A}\right)=3.2360$, which values satisfy the conditions (22). The corresponding (un-normalized) state is

$$
\begin{aligned}
\left|\psi_{1}\right\rangle= & +0.069455|000\rangle+|001\rangle+|010\rangle \\
& -0.762707|011\rangle+0.604546|100\rangle \\
& -0.475110|101\rangle-0.762707|110\rangle .
\end{aligned}
$$

The negativity — an entanglement measure [51] — of the bipartite state $\rho_{A B}$ defined by $\left|\psi_{1}\right\rangle$ in Eq. (25) and $p=1$ in Eq. (11) is given by $E_{N}=0.1517$. In Appendix A we provide Alice's optimal observables $A_{x}=\vec{a}_{x} \cdot \vec{\sigma}$ for this scenario, corresponding to the maximum value $Q\left(\rho_{A B}\right)=3.2688$ in Eq. (17). In particular, $\left|\psi_{1}\right\rangle$ in Eq. (25) through $\rho_{A B}$ defines $G_{x}$ in Eq. (19), which in turn provides the $A_{x}=\vec{a}_{x} \cdot \vec{\sigma}$ observables (21). The corresponding Bloch vectors $\vec{a}_{x}$ are given by (A2) in Appendix A, which are represented in Fig. 2. The figure shows the icosahedron configuration of Bob's unit vectors $\vec{b}_{x}$ (blue markers) and Alice's six Bloch vectors $\vec{a}_{x}$ (red markers), $x=1, \ldots, 6$. We next turn to our main problem of finding a genuine cyclic EPR-steerable state for projective measurements.

\section{Scenario 2}

We are looking for a three-qubit state in the form (11), where each two-qubit marginal $\rho_{A B}=\rho_{B C}=\rho_{C A}$ is one-way steerable considering arbitrary projective measurements. For this purpose, we will rely on the recent two-qubit steerability result of Nguyen et al. [41]. In that work the critical radius $R\left(\sigma_{A B}\right)$ of an arbitrary two-qubit state $\sigma_{A B}$ is defined, and it is shown that $\sigma_{A B}$ is unsteerable from Alice to Bob if and only if $R\left(\sigma_{A B}\right) \geqslant 1$. The critical radius has an operational meaning as well; $1-R\left(\sigma_{A B}\right)$ measures the distance from the state $\sigma_{A B}$ to the border of the unsteerable states relative to the separable state $\left(\mathbb{I}_{A} / 2\right) \otimes \sigma_{B}$, where $\sigma_{B}=\operatorname{tr}_{A}\left(\sigma_{A B}\right)$ is Bob's reduced state. Moreover, Ref. [41] gives an efficient numerical method to compute the critical radius for any two-qubit state considering arbitrary projective measurements. Namely, given a two-qubit state $\sigma_{A B}$, the numerical algorithm in Ref. [41] gives both an upper bound $R^{\text {out }}$ and a lower bound $R^{\text {in }}$ on the critical radius $R$ of the state:

$$
R^{\text {in }}\left(\sigma_{A B}\right) \leqslant R\left(\sigma_{A B}\right) \leqslant R^{\text {out }}\left(\sigma_{A B}\right) .
$$

The size of the interval above is related to a parameter $N$ of the method of Ref. [41]. This parameter defines the number of vertices $N$ of an inscribed polyhedron of the unit sphere, and in the implemented version the largest parameter is $N=$ 252 , which gives a small gap (typically smaller than $0.01 R^{\text {in }}$ ) between the upper and lower bound values in Eq. (26).

Our goal is then to find a one-way steerable state $\rho_{A B}$ for projective measurements using the numerical procedure in Ref. [41], where $\rho_{A B}=\operatorname{tr}_{C} \rho(p)$, where $\rho(p)$ defines the three-qubit state (11). We set $p=1$ and assume the most general form of the state $\left|\psi_{1}\right\rangle$ in Eq. (12). If we happen to find that for some $\left|\psi_{1}\right\rangle$ both $R\left(\rho_{A B}\right)<1$ and $R\left(\rho_{B A}\right) \geqslant 1$ are satisfied, then we have found a one-way steerable state $\rho_{A B}$, and we have achieved our goal. Formally, our task reduces to the following feasibility problem. Find a three-qubit state $\rho(p=1)$ in the form of Eq. (11) with two-qubit reduced state $\rho_{A B}$ such that

$$
\begin{aligned}
& R_{1} \equiv R^{\text {out }}\left(\rho_{A B}\right)<1, \\
& R_{2} \equiv R^{\text {in }}\left(\rho_{B A}\right) \geqslant 1 .
\end{aligned}
$$

Such inequalities entail the existence of $R\left(\rho_{A B}\right)<1$ and $R\left(\rho_{B A}\right) \geqslant 1$, thus directly proving that $\rho_{A B}$ is one-way steerable and eventually proving the existence of cyclic EPR steering for a state in Eq. (11). To find such a state, similarly to our steering scenario 1, we run the Nelder-Mead heuristic search. This time we maximize the objective value

$$
\begin{aligned}
q= & R_{2}-R_{1}-c_{1} \max \left[0, R_{1}-1\right]-c_{2} \max \left[0,1-R_{2}\right] \\
& -c_{3}\left[\mathcal{H}\left(R_{1}-1\right)+\mathcal{H}\left(1-R_{2}\right)\right]
\end{aligned}
$$

over all coefficients of $\left|\psi_{1}\right\rangle$, where we set $c_{i}$ positive, such as $c_{1}=c_{2}=2 c_{3}=1$, where $\mathcal{H}$ above denotes the Heaviside function. Note that the last term multiplied by $c_{3}$ gives an additional penalty to the objective value if the constraint $R_{1}<$ 1 or $R_{2}>1$ in Eq. (27) is not satisfied. By setting $c_{1} \neq c_{2}$ we can search for asymmetric solutions, that is, when the average value $\left(R_{1}+R_{2}\right) / 2$ tends to differ from 1 . However, the problem with this simple scheme is that the running time for a single evaluation of $q$ in Eq. (28) by the parameter $N=252$ takes about an hour on a standard desktop computer. Therefore the Nelder-Mead algorithm becomes very timeconsuming. Note that in the complex-valued case we have to optimize over $2 \times 2^{3}-1=15$ free variables defining the three-qubit pure state $\left|\psi_{1}\right\rangle$, which usually requires thousands of iterations to achieve convergence. To increase efficiency, we may choose to optimize (28) with a smaller parameter, say, $N=92$, for a couple of iteration steps and then switch to the more time-consuming $N=252$ case. In practice, we have chosen an even simpler (i.e., less time-consuming) solution for this preprocessing step. Namely, for an arbitrary two-qubit state $\sigma_{A B}$ the following analytical upper bound can be given $[41,52]$ :

$$
R\left(\sigma_{A B}\right) \leqslant 2 \pi N_{T}|\operatorname{det}(T)| \equiv R_{a}\left(\sigma_{A B}\right) .
$$

To obtain $N_{T}$ and $\operatorname{det}(T)$, first we have to bring $\sigma_{A B}$ into a so-called normal form $\tilde{\sigma}_{A B}$, for which Bob's marginal is completely random and the correlation matrix $T$ defined by $T_{i, j}=$ $\operatorname{tr}\left(\tilde{\sigma}_{A B} \sigma_{i} \otimes \sigma_{j}\right)$ for $i, j=1,2,3$ has only diagonal elements. This transformation can be found in Ref. [41]. Then the explicit expressions are as follows: $N_{T}^{-1}=\int d S(\vec{n})\left[\vec{n}^{T} T^{-2} \vec{n}\right]^{-2}$, 
where the integration runs over the surface of the Bloch sphere, and $\operatorname{det}(T)=\prod_{i=1}^{3} T_{i i}$. For Bell-diagonal states, i.e., states for which the local marginals of Alice and Bob are maximally mixed, the upper bound (29) is tight [41]. In this case, $R\left(\sigma_{A B}\right)=R\left(\sigma_{B A}\right)$, which is opposite to the property we are looking for. For a more relevant example involving the nontrivial local marginal term as well, we refer to the one-parameter family of one-way steerable two-qubit states [40,41]:

$$
\rho=\alpha\left|\psi_{\theta}\right\rangle\left\langle\psi_{\theta}\right|+(1-\alpha) \frac{\mathbb{I}_{2}}{2} \otimes \rho_{B},
$$

where $\left|\psi_{\theta}\right\rangle=\cos (\theta / 2)|00\rangle+\sin (\theta / 2)|11\rangle$ with $0 \leqslant \theta \leqslant$ $(\pi / 4)$ and $0 \leqslant \alpha \leqslant 1$. This state is steerable from Alice to Bob for $\alpha>(1 / 2)$ and $\theta>0$. However, the state is unsteerable from Bob to Alice for certain parameter ranges as shown in Refs. [40,41]. Let us choose the parameters: $\theta=\pi / 6$ and $\alpha=0.6$ for which the state is one-way steerable. Indeed, using the algorithm of Ref. [41] by $N=252$ we obtain the following upper and lower bounds for the critical radius:

$$
\begin{aligned}
R^{\text {in }}\left(\rho_{A B}\right) & =0.83166697, \\
R^{\text {out }}\left(\rho_{A B}\right) & =0.84010549, \\
R^{\text {in }}\left(\rho_{B A}\right) & =1.03284644, \\
R^{\text {out }}\left(\rho_{B A}\right) & =1.03527766 .
\end{aligned}
$$

On the other hand, the analytic upper bound values are as follows:

$$
\begin{aligned}
& R_{a}\left(\rho_{A B}\right)=0.833333333, \\
& R_{a}\left(\rho_{B A}\right)=1.626113143 .
\end{aligned}
$$

Comparing the value of (31) and 32, we see that the upper bound value is reasonably good in one direction but can perform poorly in the other direction. Still, the difference $R_{a}\left(\rho_{B A}\right)-R_{a}\left(\rho_{A B}\right)$ seems to be a good indicator of the asymmetry of the state, and we use this function as part of a preprocessing step in the optimization detailed below. Specifically, we run an unconstrained Nelder-Mead search to maximize the following expression:

$$
q=R_{a}\left(\rho_{B A}\right)-R_{a}\left(\rho_{A B}\right)-c \max \left[0, R_{a}\left(\rho_{B A}\right)-\delta\right]
$$

over $\rho_{A B}$, where the optimization variables are the coefficients of $\left|\psi_{1}\right\rangle$ in Eq. (12). Note that the last subtracted (positive) term is a penalty on the objective function whenever $R_{a}\left(\rho_{B A}\right)>\delta$. We have chosen $\delta$ to be greater than 1, in our actual computation $\delta=1.2$, since $R_{a}\left(\rho_{B A}\right)$ can be considerably larger than $R\left(\rho_{B A}\right)$, as we saw in the previous example.

The optimization (33) provides us with a state $\left|\psi_{1}\right\rangle$, which we pass to the further Nelder-Mead search (28) with parameter $N=252$. As a result of this two-step optimization procedure, we managed to obtain true one-way steerable states $\rho_{A B}$. We found the simplest state $\left|\psi_{1}\right\rangle$ defined by six real parameters (by setting $c_{2}>c_{1}$ and $c_{3}=1$ ), where the state vector $\left|\psi_{1}\right\rangle$ (up to normalization) looks as follows:

$$
\begin{aligned}
\left|\psi_{1}\right\rangle= & |000\rangle-0.321193|001\rangle-0.477021|010\rangle \\
& +0.045221|011\rangle-0.718592|100\rangle \\
& +0.213715|101\rangle-0.0482|110\rangle .
\end{aligned}
$$

Note that here the coefficient of $|000\rangle$ is set to 1 and the coefficient of $|111\rangle$ is set to zero in the course of optimization. The lower and upper bounds on the critical radius of the state $\rho_{A B}$ and its swapped state $\rho_{B A}$ are

$$
\begin{aligned}
R^{\text {in }}\left(\rho_{A B}\right) & =0.9925396, \\
R^{\text {out }}\left(\rho_{A B}\right) & =0.99822006, \\
R^{\text {in }}\left(\rho_{B A}\right) & =1.0000028, \\
R^{\text {out }}\left(\rho_{B A}\right) & =1.0065179 .
\end{aligned}
$$

Since $R^{\text {out }}\left(\rho_{A B}\right)<1<R^{\text {in }}\left(\rho_{B A}\right)$ holds in the above, our state $\rho(p=1)$ with $\left|\psi_{1}\right\rangle$ in Eq. (34) indeed defines a feasible solution to the phenomenon of cyclic EPR steering. Also by direct computation, the entanglement negativity of $\rho_{A B}$ is given by 0.0630 , which is to be compared with the negativity $1 / 2$ of the maximally entangled singlet state (1). Let us define the gap

$$
\Delta\left(\rho_{A B}\right)=R^{\text {in }}\left(\rho_{B A}\right)-R^{\text {out }}\left(\rho_{A B}\right)
$$

between the feasible limits $R^{\text {in }}\left(\rho_{B A}\right) \geqslant 1$ and $R^{\text {out }}\left(\rho_{A B}\right)<1$. Note that the above example $\rho(p=1)$ with $\left|\psi_{1}\right\rangle$ in Eq. (34) does not provide the largest possible gap. In fact, we have found states with larger gaps $\Delta\left(\rho_{A B}\right)$ among generic realvalued states $\left|\psi_{1}\right\rangle$ (that is, when $\alpha_{111}$ is not set to zero), and states with even larger gaps by optimizing over complexvalued states $\left|\psi_{1}\right\rangle$. Such examples with larger $\Delta\left(\rho_{A B}\right)$ values are discussed in detail in Appendix B, with the multipartite entanglement property of the states discussed in Appendix $\mathrm{C}$.

Let us point out that the nonzero gap $\left[1-R^{\text {out }}\left(\rho_{A B}\right)\right]$ in Eq. (35) implies a whole family of feasible states for cyclic EPR steering. To this end, we set $p=0.999$ in the oneparameter family of states $\rho(p)$ defined by (11). In this case, we have the two-qubit reduced state

$$
\rho_{A B}(p)=p \rho_{A B}+(1-p) \mathbb{I}_{4} / 4,
$$

where $p=0.999$. Running the code of Ref. [41] with $N=$ 252 for $\rho_{A B}(0.999)$ and $\rho_{B A}(0.999)$ gives the following bounds on the critical radius:

$$
\begin{aligned}
R^{\text {out }}\left[\rho_{A B}(0.999)\right] & =0.99994147, \\
R^{\text {in }}\left[\rho_{B A}(0.999)\right] & =1.0030571,
\end{aligned}
$$

fulfilling both conditions in Eq. (27), which implies that $\rho_{A B}(0.999)$ is one-way steerable.

Let us now show that $\rho_{A B}(p)$ is one-way steerable not only for $p=1$ and $p=0.999$ but also for any intermediate $p \in$ $[0.999,1]$. To do this, we use the following relation. Given an arbitrary two-qubit state $\sigma$, we obtain the following inequality for any weight $q$ :

$$
R(\sigma) \leqslant R\left(q \sigma+(1-q) \mathbb{I}_{4} / 4\right),
$$

which follows from the definition of the $R$ critical radius. Indeed, by definition $r_{\text {crit }} \sigma+\left(1-r_{\text {crit }}\right) \mathbb{I}_{2} / 2 \otimes \sigma_{B}$ is on the border of unsteerable states, where $r_{\text {crit }} \equiv R(\sigma)$. However, if some isotropic noise $\mathbb{I}_{4} / 4$ is added to $\sigma$, the state cannot become steerable due to the convexity of the set of unsteerable states. Therefore the critical radius of this noisy state cannot be less than that of $\sigma$ as expressed by (39). 
Now let $\rho_{A B}(p)=\sigma$ and $1-q \equiv \epsilon>0$ in Eq. (39). Then we have $R\left[\rho_{A B}(p)\right] \leqslant R\left[\rho_{A B}[p(1-\epsilon)]\right]$. That is, $R\left[\rho_{A B}(p)\right]$ is a monotone decreasing function of $p$. By similar arguments, $R\left[\rho_{B A}(p)\right]$ is also a monotone decreasing function of $p$. Then we have that

$$
\begin{aligned}
& R\left[\rho_{A B}(p)\right] \leqslant R\left[\rho_{A B}(0.999)\right], \\
& R\left[\rho_{B A}(p)\right] \geqslant R\left[\rho_{B A}(1)\right]
\end{aligned}
$$

for any $p \in[0.999,1]$. Then our claim follows about the oneway steerability of $\rho_{A B}(p)$ in this range, which in turn implies that the family of states (11) with $\left|\psi_{1}\right\rangle$ in Eq. (34) exhibits cyclic EPR steering in the range $0.999 \leqslant p \leqslant 1$.

\section{CYCLIC STEERING WITH POVMS AND HIGHER DIMENSIONAL STATES}

Here, we show that if we do not restrict the local dimension to a qubit, we can strengthen our findings by generalizing cyclic EPR steering from projective measurements to general POVMs. To this end, we consider another construction, where the tripartite state is given by

$$
\rho_{\tilde{A} \tilde{B} \tilde{C}}=\rho_{A B} \otimes \rho_{B^{\prime} C} \otimes \rho_{C^{\prime} A^{\prime}},
$$

where the subsystems $\tilde{A}=A A^{\prime}, \tilde{B}=B B^{\prime}$, and $\tilde{C}=C C^{\prime}$ belong to Alice, Bob, and Charlie, respectively. Let us further distribute the same states between the parties, that is, $\rho_{A B}=$ $\rho_{B^{\prime} C}=\rho_{C^{\prime} A^{\prime}}$. Furthermore, let $\rho_{A B} \in L\left(\mathbb{C}^{d_{A}} \otimes \mathbb{C}^{d_{B}}\right)$ for some $d_{A}$ and $d_{B}$ be such that Alice can steer Bob's state using POVM measurements, but not the other way around (i.e., the state is one-way steerable for the most general POVMs). The existence of such a bipartite state with $d_{A}=2$ and $d_{B}=3$ was proven in Ref. [19]. More recently, the required dimension has been further reduced to $d_{A}=d_{B}=2$ in Ref. [40]. In this case, the $(d \times d \times d)$-dimensional state $\rho_{\tilde{A} \tilde{B} \tilde{C}}$ with $d=d_{A} \times d_{B}=4$ exhibits the desired cyclic EPR-steering property $A \rightarrow B$, $B \rightarrow C$, and $C \rightarrow A$.

Indeed, in order for Alice to steer Bob (and also for Bob to steer Charlie and Charlie to steer Alice), the respective parties perform measurements on their entangled parts of subsystems.

On the other hand, the unsteerability property in the opposite direction follows from the fact that the composite state $\rho_{\tilde{A} \tilde{B} \tilde{C}}$ in Eq. (41) is a product across the bipartitions $A\left|A^{\prime}, B\right| B^{\prime}$, and $C \mid C^{\prime}$. Hence we have the reduced state $\rho_{\tilde{A} \tilde{B}}=\operatorname{tr}_{\tilde{C}} \rho_{\tilde{A} \tilde{B} \tilde{C}}=$ $\rho_{A B} \otimes \rho_{A^{\prime}} \otimes \rho_{B^{\prime}}$, where $\rho_{A^{\prime}}$ and $\rho_{B^{\prime}}$ are the reduced states of $\rho_{C A^{\prime}}$ and $\rho_{B^{\prime} C}$, respectively. Due to convexity, if the state $\rho_{A B}$ admits a LHS model from Bob to Alice for POVM measurements, then the state $\rho_{\tilde{A} \tilde{B}}$ still admits a LHS model for POVM measurements.

As mentioned above, using the one-way steerable state $\rho_{A B} \in L\left(\mathbb{C}^{2} \otimes \mathbb{C}^{2}\right)$ from Ref. [40], we have a three-party cyclic EPR-steerable state (41) for POVM measurements with dimension $(4 \times 4 \times 4)$. We state it as an open problem to find a cyclic EPR-steerable state for POVMs in lower dimensions.

\section{DISCUSSION}

We have shown the existence of three-party states arranged in a triangular configuration, where each two-party reduced state is steerable in one (e.g., clockwise) direction, but unsteerable in the other (e.g., anticlockwise) direction. That is, by choosing any pair of systems out of the tripartite system belonging to parties $P$ and $P^{\prime}$, steering can occur from party $P$ to party $P^{\prime}$ if and only if $P^{\prime}$ lies clockwise of $P$. This shows a peculiar directional feature of EPR quantum correlations, which can appear neither in the phenomenon of entanglement nor in Bell nonlocality. To study this directional or handedness property of quantum correlations, we have focused mainly on three-qubit states with projective measurements. However, we also discuss the case of higher-dimensional systems and the role of POVM measurements. A couple of questions have been left open. One such question is whether our three-qubit cyclic steering result valid for projective measurements could also be extended to the most general form of POVM measurements. Second, is it true that cyclic steering for three-party systems always entails genuine tripartite entanglement? Third, it would also be interesting to generalize the construction of three-qubit translationally invariant states beyond three parties. From a more applied point of view, it would be interesting to find useful information applications of the directional feature of quantum correlations. Finally, a fully analytical proof of the three-qubit cyclic EPR-steering phenomenon would be welcome.

\section{ACKNOWLEDGMENTS}

We thank Wiesław Laskowski, Miguel Navascués, Géza Tóth, and Jordi Tura for valuable discussions. E.B. and T.V. acknowledge the support of the EU (QuantERA eDICT) and the National Research, Development and Innovation Office NKFIH (Grant No. 2019-2.1.7-ERA-NET-2020-00003).

\section{APPENDIX A: MEASUREMENT SETTINGS OF ALICE AND BOB IN SETUP 1}

Let the following six unit vectors $\vec{b}_{x}(x=1, \ldots, 6)$ correspond to Bob's measurement settings with outcome $b=0$ and their antipodal points $-\vec{b}_{x}$ correspond to the outcome $b=1$ :

$$
\begin{array}{ll}
\vec{b}_{1}=[0,1, \phi] / n, & \vec{b}_{2}=[0,1,-\phi] / n, \\
\vec{b}_{3}=[1, \phi, 0] / n, & \vec{b}_{4}=[1,-\phi, 0] / n, \\
\vec{b}_{5}=[\phi, 0,1] / n, & \vec{b}_{6}=[\phi, 0,-1] / n,
\end{array}
$$

where $\phi=(1+\sqrt{5}) / 2$ is the golden ratio and $n=\sqrt{1+\phi^{2}}$ is a normalization constant. In particular, the $\pm \vec{b}_{x}$ vectors above form the vertices of a regular icosahedron. See also Fig. 2 for the arrangement of the $\vec{b}_{x}$ vectors on the Bloch sphere (the respective endpoints are marked by small blue circles).

On the other hand, Alice's Bloch vectors $\vec{a}_{x}$ corresponding to the outcome $a=0$ look as follows:

$$
\begin{aligned}
& \vec{a}_{1}=[0.1,0.7664,-0.6346], \\
& \vec{a}_{2}=[-0.1,0.7664,0.6346], \\
& \vec{a}_{3}=[0.4959,0.8683,0.0099], \\
& \vec{a}_{4}=[0.4959,-0.8683,0.0099], \\
& \vec{a}_{5}=[0.9563,0,-0.2925], \\
& \vec{a}_{6}=[0,0,0] .
\end{aligned}
$$


TABLE I. Coefficients $\alpha_{i j k}$ of $\left|\psi_{1}^{(i)}\right\rangle$ in Eq. (12) for $i=1,2,3$.

\begin{tabular}{cccc}
\hline \hline$\alpha_{i j k}$ & $\left|\psi_{1}^{(1)}\right\rangle$ & $\left|\psi_{1}^{(2)}\right\rangle$ & $\left|\psi_{1}^{(3)}\right\rangle$ \\
\hline$\alpha_{000}$ & 1 & 1 & 1 \\
$\alpha_{001}$ & -0.321193 & -0.259910 & $-0.252592-0.065698 \mathrm{i}$ \\
$\alpha_{010}$ & -0.477021 & -0.591007 & $0.002913-0.000635 \mathrm{i}$ \\
$\alpha_{011}$ & 0.045221 & 0.028007 & $0.025469+0.025479 \mathrm{i}$ \\
$\alpha_{100}$ & -0.718592 & -0.798924 & $-0.120348-0.110323 \mathrm{i}$ \\
$\alpha_{101}$ & 0.213715 & 0.206125 & $-0.103340-0.161335 \mathrm{i}$ \\
$\alpha_{110}$ & -0.0482 & -0.079214 & $-0.044067-0.089806 \mathrm{i}$ \\
$\alpha_{111}$ & 0 & -0.000311 & $0.055929-0.044192 \mathrm{i}$ \\
\hline \hline
\end{tabular}

In Fig. 2, the endpoints of the six $\vec{a}_{x}$ vectors are denoted by small red circles.

\section{APPENDIX B: NUMERICALLY OBTAINED CYCLIC EPR-STEERABLE STATES IN SETUP 2}

We define the state $\left|\psi_{1}\right\rangle=\sum_{i, j, k=0}^{1} \alpha_{i j k}|i j k\rangle$ according to Eq. (12), which is used to define the state $\rho(p)$ in Eq. (11). Here, we set $p=1$, so we simply have

$$
\rho_{A B C} \equiv \rho(1)=\frac{\sum_{i=1}^{3}\left|\psi_{i}\right\rangle\left\langle\psi_{i}\right|}{3},
$$

where $\left|\psi_{2}\right\rangle$ and $\left|\psi_{3}\right\rangle$ are derived from one- and two-qubit translations of $\left|\psi_{1}\right\rangle$ according to Eq. (8). Note that the reduced two-qubit states of $\rho_{A B C}$ above have the properties $\rho_{A B}=\rho_{B C}=\rho_{C A}$. Therefore, if we find that $\rho_{A B}$ is one-way steerable, then it also holds true for the other two marginals $\rho_{B C}$ and $\rho_{C A}$, which implies the existence of a tripartite cyclic EPR-steerable state. We use the metric $\Delta\left(\rho_{A B}\right)=R^{\text {in }}\left(\rho_{B A}\right)-$ $R^{\text {out }}\left(\rho_{A B}\right)$ to the quality of the cyclic steerability property of the state (B1), where the values of the feasible critical radii are restricted to $R^{\text {in }}\left(\rho_{B A}\right) \geqslant 1$ and $R^{\text {out }}\left(\rho_{A B}\right)<1$. Then according to the conditions (27), $\Delta\left(\rho_{A B}\right)>0$ implies that $\rho_{A B}$ is one-way steerable for projective measurements, and this in turn implies that $\rho_{A B C}$ is a cyclically steerable state. In what follows, we present three such states, denoted $\rho_{A B C}^{(i)}$, where each state is defined by $\left|\psi_{1}^{(i)}\right\rangle$ for $i=1,2,3$ in Eq. (B1).

Our first state $\rho_{A B C}^{(1)}$ corresponds to the state (34) in the main text. The coefficients $\alpha_{i j k}$ of this state are also shown in the second column of Table I. Here, we set $c_{111}=0$, and the other seven coefficients are real valued. The corresponding reduced two-qubit state $\rho_{A B}^{(1)}$ (and its swapped state $\rho_{B A}^{(1)}$ ) has the following critical radius parameters:

$$
\begin{aligned}
R^{\text {out }}\left(\rho_{A B}^{(1)}\right) & =0.99822006, \\
R^{\text {in }}\left(\rho_{B A}^{(1)}\right) & =1.0000028,
\end{aligned}
$$

resulting in $\Delta\left(\rho_{A B}^{(1)}\right)=0.00178274$. By direct calculation, the negativity of this state is $E_{N}=0.0630$.

Our next state $\rho_{A B C}^{(2)}$ corresponds to $\left|\psi_{1}^{(2)}\right\rangle$ in Table I (third column). This is the best real-valued solution we have found, which has the following critical radius parameters:

$$
\begin{aligned}
R^{\text {out }}\left(\rho_{A B}^{(2)}\right) & =0.99999963, \\
R^{\text {in }}\left(\rho_{B A}^{(2)}\right) & =1.00237752,
\end{aligned}
$$

with $\Delta\left(\rho_{A B}^{(2)}\right)=0.00237789$. On the other hand, the negativity of this state is $E_{N}=0.0679$.

Finally, the best complex-valued solution corresponding to the state $\left|\psi_{1}^{(3)}\right\rangle$ is shown in the fourth column of Table I. This solution has the following critical radius parameters:

$$
\begin{aligned}
R^{\text {out }}\left(\rho_{A B}^{(3)}\right) & =0.99999898, \\
R^{\text {in }}\left(\rho_{B A}^{(3)}\right) & =1.0028066,
\end{aligned}
$$

with $\Delta\left(\rho_{A B}^{(3)}\right)=0.00280762$. The negativity of this two-qubit state is $E_{N}=0.0600$.

\section{APPENDIX C: ENTANGLEMENT PROPERTIES OF THE NUMERICALLY OBTAINED CYCLIC EPR-STEERABLE STATES IN SCENARIO 2}

Here, we analyze the tripartite entanglement properties of the cyclic EPR-steerable states $\rho_{A B C}^{(i)}, i=1,2,3$, given in Appendix $B$. In particular, we use the criteria of genuine tripartite entanglement (GTE) developed by Gühne and Seevinck [53] and the numerical method developed in Ref. [54]. On the one hand, we have already given the negativity $E_{N}$ of the two-qubit reduced states of $\rho_{A B C}^{(i)}$ in Appendix B. Since all $E_{N}\left(\rho_{A B}^{(i)}\right)$ are strictly positive, it entails that all two-qubit marginals of $\rho_{A B C}^{(i)}$ are entangled. However, to the best of our knowledge, it is an open problem whether a given threequbit state with all two-qubit marginals entangled implies that the three-qubit state is genuinely tripartite entangled (GTE). Indeed, multipartite entanglement properties can be very complex even for simple multipartite states, such as three-qubit states $[1,2,45]$.

Here, we show that the complex-valued three-qubit state $\rho_{A B C}^{(3)}$ given in Appendix B is genuinely tripartite entangled. To this end, we first define biseparable states. A three-party state $\rho_{A B C}$ can be written in biseparable form if and only if

$$
\rho_{A B C}=p \rho_{A \mid B C}+q \rho_{B \mid A C}+(1-p-q) \rho_{C \mid A B},
$$

where $p, q,(1-p-q)$ are non-negative numbers and $\rho_{A \mid B C}$ represents any tripartite biseparable state with respect to the cut $A \mid B C$ (and the other terms are defined similarly). If $\rho_{A B C}$ cannot be written in this form, we say that it is GTE.

We then show that the three-qubit state $\rho_{A B C}^{(3)}$ is GTE by invoking the following sufficient criterion of GTE [53]:

$$
\left|\rho_{1,8}\right|>\sqrt{\rho_{2,2} \rho_{7,7}}+\sqrt{\rho_{3,3} \rho_{6,6}}+\sqrt{\rho_{4,4} \rho_{5,5}},
$$

where $\rho_{i, j}$ denotes the $(i, j)$ entry of a three-qubit density matrix $\rho$, where the standard product basis $\{|000\rangle,|001\rangle, \ldots,|111\rangle\}$ is assumed. Since for our particular state $\rho_{A B C}^{(3)},\left|\rho_{1,8}\right|=0.0621$ and the right-hand side of $(\mathrm{C} 2)$ is 0.0588 , the criterion is readily satisfied. Therefore our state is GTE, as reported.

Note also that we could not detect GTE for either $\rho_{A B C}^{(1)}$ or $\rho_{A B C}^{(2)}$ using the criteria of Ref. [53]. However, the numerical method of Ref. [54] can detect GTE in these states as well. We then leave it as an open problem whether there exist three-party cyclically steerable states which are not GTE. In this respect, it is worth noting that for higher-dimensional tripartite systems there exist biseparable states [i.e., states that 
can be written in the form (C1)] in which all reduced bipartite systems are entangled. Such an example was presented in
Ref. [55]. It remains to be proven, however, whether these states exhibit cyclic EPR steering.
[1] R. Horodecki, P. Horodecki, M. Horodecki, and K. Horodecki, Quantum entanglement, Rev. Mod. Phys. 81, 865 (2009).

[2] O. Gühne and G. Tóth, Entanglement detection, Phys. Rep. 474, 1 (2009).

[3] A. Einstein, B. Podolsky, and N. Rosen, Can quantummechanical description of physical reality be considered complete? Phys. Rev. 47, 777 (1935).

[4] J. Bell, On the Einstein Podolsky Rosen paradox, Phys. Phys. Fiz. 1, 195 (1964).

[5] N. Brunner, D. Cavalcanti, S. Pironio, V. Scarani, and S. Wehner, Bell nonlocality, Rev. Mod. Phys. 86, 419 (2014); 86, 839(E) (2014).

[6] V. Scarani, Bell Nonlocality (Oxford University Press, Oxford, 2019).

[7] E. Schrödinger, Discussion of probability relations between separated systems, Proc. Cambridge Philos. Soc. 31, 555 (1935).

[8] D. Cavalcanti and P. Skrzypczyk, Quantum steering: a review with focus on semidefinite programming, Rep. Prog. Phys. 80, 024001 (2017).

[9] R. Uola, A. C. S. Costa, H. C. Nguyen, and O. Gühne, Quantum steering, Rev. Mod. Phys. 92, 015001 (2020).

[10] M. D. Reid, Demonstration of the Einstein-PodolskyRosen paradox using nondegenerate parametric amplification, Phys. Rev. A 40, 913 (1989).

[11] M. D. Reid, P. D. Drummond, W. P. Bowen, E. G. Cavalcanti, P. K. Lam, H. A. Bachor, U. L. Andersen, and G. Leuchs, Colloquium: the Einstein-Podolsky-Rosen paradox: From concepts to applications, Rev. Mod. Phys. 81, 1727 (2009).

[12] H. M. Wiseman, S. J. Jones, and A. C. Doherty, Steering, Entanglement, Nonlocality, and the Einstein-Podolsky-Rosen Paradox, Phys. Rev. Lett. 98, 140402 (2007).

[13] D. J. Saunders, S. J. Jones, H. M. Wiseman, and G. J. Pryde, Experimental EPR-steering using Bell-local states, Nat. Phys. 6, 845 (2010).

[14] R. F. Werner, Quantum states with Einstein-Podolsky-Rosen correlations admitting a hidden-variable model, Phys. Rev. A 40, 4277 (1989).

[15] A. Peres, Separability Criterion for Density Matrices, Phys. Rev. Lett. 77, 1413 (1996).

[16] P. Diviánszky, E. Bene, and T. Vértesi, Qutrit witness from the Grothendieck constant of order four, Phys. Rev. A 96, 012113 (2017).

[17] F. Hirsch, M. T. Quintino, T. Vértesi, M. Navascués, and N. Brunner, Better local hidden variable models for two-qubit Werner states and an upper bound on the Grothendieck constant $K_{G}(3)$, Quantum 1, 3 (2017).

[18] A. Acín, N. Gisin, and B. Toner, Grothendieck's constant and local models for noisy entangled quantum states, Phys. Rev. A 73, 062105 (2006).

[19] M. T. Quintino, T. Vértesi, D. Cavalcanti, R. Augusiak, M. Demianowicz, A. Acín, and N. Brunner, Inequivalence of entanglement, steering, and Bell nonlocality for general measurements, Phys. Rev. A 92, 032107 (2015).
[20] C. Branciard, E. G. Cavalcanti, S. P. Walborn, V. Scarani, and H. M. Wiseman, One-sided device-independent quantum key distribution: Security, feasibility, and the connection with steering, Phys. Rev. A 85, 010301(R) (2012).

[21] E. Kaur, M. M. Wilde, and A. Winter, Fundamental limits on key rates in device-independent quantum key distribution, New J. Phys. 22, 023039 (2020).

[22] P. Skrzypczyk and D. Cavalcanti, Maximal Randomness Generation from Steering Inequality Violations Using Qudits, Phys. Rev. Lett. 120, 260401 (2018).

[23] Y. Z. Law, L. P. Thinh, J.-D. Bancal, and V. Scarani, Quantum randomness extraction for various levels of characterization of the devices, J. Phys. A: Math. Theor. 47, 424028 (2014).

[24] F. J. Curchod, M. Johansson, R. Augusiak, M. J. Hoban, P. Wittek, and A. Acín, Unbounded randomness certification using sequences of measurements, Phys. Rev. A 95, 020102(R) (2017).

[25] M. Piani and J. Watrous, Necessary and Sufficient Quantum Information Characterization of Einstein-Podolsky-Rosen Steering, Phys. Rev. Lett. 114, 060404 (2015).

[26] B. Yadin, M. Fadel, and M. Gessner, Metrological complementarity reveals the Einstein-Podolsky-Rosen paradox, Nat. Commun. 12, 2410 (2021).

[27] B. Wittmann, S. Ramelow, F. Steinlechner, N. K. Langford, N. Brunner, H. M. Wiseman, R. Ursin, and A. Zeilinger, Loophole-free Einstein-Podolsky-Rosen experiment via quantum steering, New J. Phys. 14, 053030 (2012).

[28] A. J. Bennet, D. A. Evans, D. J. Saunders, C. Branciard, E. G. Cavalcanti, H. M. Wiseman, and G. J. Pryde, Arbitrarily Loss-Tolerant Einstein-Podolsky-Rosen Steering Allowing a Demonstration over $1 \mathrm{~km}$ of Optical Fiber with No Detection Loophole, Phys. Rev. X 2, 031003 (2012).

[29] D. H. Smith, G. Gillett, M. P. de Almeida, C. Branciard, A. Fedrizzi, T. J. Weinhold, A. Lita, B. Calkins, T. Gerrits, H. M. Wiseman, S. W. Nam, and A. G. White, Conclusive quantum steering with superconducting transition-edge sensors, Nat. Commun. 3, 625 (2012).

[30] Q. Y. He, M. D. Reid, T. G. Vaughan, C. Gross, M. Oberthaler, and P. D. Drummond, Einstein-Podolsky-Rosen Entanglement Strategies in Two-Well Bose-Einstein Condensates, Phys. Rev. Lett. 106, 120405 (2011).

[31] M. Fadel, T. Zibold, B. Décamps, and P. Treutlein, Spatial entanglement patterns and Einstein-Podolsky-Rosen steering in Bose-Einstein condensates, Science 360, 409 (2018).

[32] P. Kunkel, M. Prüfer, H. Strobel, D. Linnemann, A. Frölian, T. Gasenzer, M. Gärttner, and M. K. Oberthaler, Spatially distributed multipartite entanglement enables EPR steering of atomic clouds, Science 360, 413 (2018).

[33] Q. Y. He and M. D. Reid, Towards an Einstein-Podolsky-Rosen paradox between two macroscopic atomic ensembles at room temperature, New J. Phys. 15, 063027 (2013).

[34] M. M. Weston, S. Slussarenko, H. M. Chrzanowski, S. Wollmann, L. K. Shalm, V. B. Verma, M. S. Allman, S. W. Nam, and G. J. Pryde, Heralded quantum steering over a high-loss channel, Sci. Adv. 4, e1701230 (2018). 
[35] B. Hensen, H. Bernien, A. E. Dréau, A. Reiserer, N. Kalb, M. S. Blok, J. Ruitenberg, R. F. L. Vermeulen, R. N. Schouten, C. Abellán, W. Amaya, V. Pruneri, M. W. Mitchell, M. Markham, D. J. Twitchen, D. Elkouss, S. Wehner, T. H. Taminiau, and R. Hanson, Loophole-free Bell inequality violation using electron spins separated by 1.3 kilometres, Nature (London) 526, 682 (2015); see also M. Giustina, M. A. M. Versteegh, S. Wengerowsky, J. Handsteiner, A. Hochrainer, K. Phelan, F. Steinlechner, J. Kofler, J. A. Larsson, C. Abellan, W. Amaya, V. Pruneri, M. W. Mitchell, J. Beyer, T. Gerrits, A. E. Lita, L. K. Shalm, S. W. Nam, T. Scheidl, R. Ursin et al., SignificantLoophole-Free Test of Bell's Theorem with Entangled Photons, Phys. Rev. Lett. 115, 250401 (2015); L. K. Shalm, E. MeyerScott, B. G. Christensen, P. Bierhorst, M. A. Wayne, M. J. Stevens, T. Gerrits, S. Glancy, D. R. Hamel, M. S. Allman, K. J. Coakley, S. D. Dyer, C. Hodge, A. E. Lita, V. B. Verma, C. Lambrocco, E. Tortorici, A. L. Migdall, Y. Zhang, D. R. Kumor et al., Strong Loophole-Free Test of Local Realism, ibid. 115, 250402 (2015).

[36] J. Bowles, T. Vértesi, M. T. Quintino, and N. Brunner, Oneway Einstein-Podolsky-Rosen Steering, Phys. Rev. Lett. 112, 200402 (2014).

[37] S. L. W. Midgley, A. J. Ferris, and M. K. Olsen, Asymmetric Gaussian steering: When Alice and Bob disagree, Phys. Rev. A 81, 022101 (2010).

[38] M. K. Olsen, Asymmetric Gaussian harmonic steering in second-harmonic generation, Phys. Rev. A 88, 051802(R) (2013).

[39] P. Skrzypczyk, M. Navascués, and D. Cavalcanti, Quantifying Einstein-Podolsky-Rosen Steering, Phys. Rev. Lett. 112, 180404 (2014).

[40] J. Bowles, F. Hirsch, M. T. Quintino, and N. Brunner, Sufficient criterion for guaranteeing that a two-qubit state is unsteerable, Phys. Rev. A 93, 022121 (2016).

[41] H. C. Nguyen, H.-V. Nguyen, and O. Gühne, Geometry of Einstein-Podolsky-Rosen Correlations, Phys. Rev. Lett. 122, 240401 (2019).

[42] V. Händchen, T. Eberle, S. Steinlechner, A. Samblowski, T. Franz, R. F. Werner, and R. Schnabel, Observation of one-way Einstein-Podolsky-Rosen steering, Nat. Photon. 6, 596 (2012).

[43] S. Wollmann, N. Walk, A. J. Bennet, H. M. Wiseman, and G. J. Pryde, Observation of Genuine One-Way Einstein-PodolskyRosen Steering, Phys. Rev. Lett. 116, 160403 (2016); see also K. Sun, X. J. Ye, J. S. Xu, X. Y. Xu, J. S. Tang, Y. C. Wu, J. L. Chen, C. F. Li, and G. C. Guo, Experimental Quantification of Asymmetric Einstein-Podolsky-Rosen Steering, ibid. 116,
160404 (2016); Y. Xiao, X. J. Ye, K. Sun, J. S. Xu, C. F. Li, and G. C. Guo, Demonstration of Multisetting One-Way Einstein-Podolsky-Rosen Steering in Two-Qubit Systems, ibid. 118, 140404 (2017); N. Tischler, F. Ghafari, T. J. Baker, S. Slussarenko, R. B. Patel, M. M. Weston, S. Wollmann, L. K. Shalm, V. B. Verma, S. W. Nam, H. C. Nguyen, H. M. Wiseman, and G. J. Pryde, Conclusive Experimental Demonstration of One-Way Einstein-Podolsky-Rosen Steering, ibid. 121, 100401 (2018).

[44] E. G. Cavalcanti, Q. Y. He, M. D. Reid, and H. M. Wiseman, Unified criteria for multipartite quantum nonlocality, Phys. Rev. A 84, 032115 (2011).

[45] S. Szalay, Multipartite entanglement measures, Phys. Rev. A 92, 042329 (2015)

[46] D. Cavalcanti, P. Skrzypczyk, G. H. Aguilar, R. V. Nery, P. H. Souto Ribeiro, and S. P. Walborn, Detection of entanglement in asymmetric quantum networks and multipartite quantum steering, Nat. Commun. 6, 7941 (2015).

[47] A. Milne, S. Jevtic, D. Jennings, H. Wiseman, and T. Rudolph, Quantum steering ellipsoids, extremal physical states and monogamy, New J. Phys. 16, 083017 (2014); 17, 019501(E) (2015); S. Cheng, A. Milne, M. J. W. Hall, and H. M. Wiseman, Volume monogamy of quantum steering ellipsoids for multiqubit systems, Phys. Rev. A 94, 042105 (2016).

[48] W. Dur, G. Vidal, and J. I. Cirac, Three qubits can be entangled in two inequivalent ways, Phys. Rev. A 62, 062314 (2000).

[49] D. M. Greenberger, M. A. Horne, and A. Zeilinger, Going beyond Bell's theorem, in Bell's Theorem, Quantum Theory, and Conceptions of the Universe, edited by M. Kafatos (Kluwer Academic, Dordrecht, 1989), pp. 69-72.

[50] J. A. Nelder and R. Mead, A simplex method for function minimization, Comput. J. 7, 308 (1965).

[51] G. Vidal and R. F. Werner, Computable measure of entanglement, Phys. Rev. A 65, 032314 (2002).

[52] S. Jevtic, M. J. W. Hall, M. R. Anderson, M. Zwierz, and H. M. Wiseman, Einstein-Podolsky-Rosen steering and the steering ellipsoid, J. Opt. Soc. Am. B 32, A40 (2015).

[53] O. Gühne and M. Seevinck, Separability criteria for genuine multiparticle entanglement, New J. Phys. 12, 053002 (2010).

[54] B. Jungnitsch, T. Moroder, and O. Gühne, Taming Multiparticle Entanglement, Phys. Rev. Lett. 106, 190502 (2011).

[55] N. Brunner and T. Vértesi, Persistency of entanglement and nonlocality in multipartite quantum systems, Phys. Rev. A 86, 042113 (2012). 